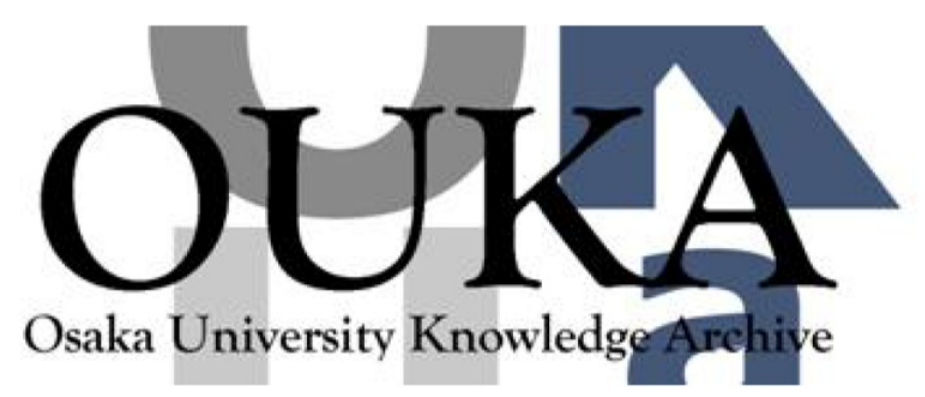

\begin{tabular}{|c|l|}
\hline Title & $\begin{array}{l}\text { Fabrication of nanoscale gaps using a } \\
\text { combination of self-assembled mo lecular and } \\
\text { electron beam lithographic techniques }\end{array}$ \\
\hline Author(s) & Negishi, R; Hasegawa, T; Terabe, K et al. \\
\hline Citation & $\begin{array}{l}\text { APPLIED PHYSICS LETTERS. 88(22) p. 223111-1- } \\
\text { p. 223111-3 }\end{array}$ \\
\hline Issue Date & 2006-05-29 \\
\hline oaire:version & VoR \\
\hline URL & https://hdl. handle. net/11094/3175 \\
\hline rights & \\
\hline Note & \\
\hline
\end{tabular}

Osaka University Knowledge Archive : OUKA

https://ir. Library. osaka-u. ac. jp/

Osaka University 


\title{
Fabrication of nanoscale gaps using a combination of self-assembled molecular and electron beam lithographic techniques
}

\author{
R. Negishi, ${ }^{a)}$ T. Hasegawa, K. Terabe, and M. Aono \\ Nanoscale Quantum Conductor Array Project, ICORP, JST, Kawaguchi, Saitama 332-0112, Japan \\ and Nanomaterials Laboratories, National Institute for Materials Science (NIMS), 1-1 Namiki, Tsukuba, \\ Ibaraki 305-0044, Japan \\ T. Ebihara \\ Nanomaterials Laboratories, NIMS, 1-1 Namiki, Tsukuba, Ibaraki 305-0044, Japan \\ H. Tanaka and T. Ogawa \\ Research Center for Molecular-Scale Nanoscience, Institute for Molecular Science, 5-1 Higashiyama, \\ Myodaiji, Okazaki, Aichi 444-8787, Japan
}

(Received 18 January 2006; accepted 19 April 2006; published online 2 June 2006)

\begin{abstract}
We have developed and tested a new method of fabricating nanogaps using a combination of self-assembled molecular and electron beam lithographic techniques. The method enables us to control the gap size with an accuracy of approximately $2 \mathrm{~nm}$ and designate the positions where the nanogaps should be formed with high-resolution patterning by using electron beam lithography. We have demonstrated the utility of the fabricated nanogaps by measuring a single electron tunneling phenomenon through dodecanethiol-coated $\mathrm{Au}$ nanoparticles placed in the fabricated nanogap. (C) 2006 American Institute of Physics. [DOI: 10.1063/1.2209208]
\end{abstract}

Fabrication of nanoscale gaps between two metal electrodes has recently attracted considerable attention because these nanogaps enable us to measure the electronic transport properties of nanocrystals ${ }^{1,2}$ and molecules ${ }^{3,4}$ that exhibit quantum effects due to the electron confinements. Moreover, nanofabrication techniques have great potential for achieving a breakthrough in making functional devices. ${ }^{5}$ Therefore, substantial efforts have been made to fabricate nanogaps using a variety of techniques, such as electron beam lithography (EBL), ${ }^{2,6}$ shadow evaporation, ${ }^{7,8}$ electromigration, ${ }^{9}$ electroplating, ${ }^{10,11}$ and break junction. ${ }^{12}$ Although all of these techniques have achieved the formation of sub-10-nm gaps, it is difficult to control the position and the size of the gap to the range of sub-2-nm, which corresponds to the general size of single molecules.

The nanolithographic technique using molecular rulers ${ }^{13-16}$ has a great advantage for maintaining the gap size in the range of sub-2-nm because the gap size is well defined by the multilayer films composed of multiple organic molecules and metal ion layers (the thickness of the multilayer films as a molecular ruler is sub-2-nm per layer). Recently, McCarty reported a method of controlling the size and the position of the nanogaps using a combination of conventional photolithographic and self-assembled molecular (SAM) lithographic techniques. ${ }^{17}$ Although the method allows us to make nanogaps with selected positions, the resolutions of the position and the width of the gap electrodes made using this method are limited to approximately $1 \mu \mathrm{m}$ because it uses photolithography.

To measure electronic transport properties through a single nanostructure or a single molecule between the nanogap electrodes, not only the nanoscale gap size but also nanoscale width of the electrodes is highly desirable to avoid

\footnotetext{
a) Author to whom correspondence should be addressed; electronic mail:
} NEGISHI.Ryota@nims.go.jp complicating the nanostructures or the molecular assembly at the gap region.

We have developed a new method of fabricating nanogaps using a combination of EBL and the SAM lithographic techniques. Using EBL enables us to control the position and the width of the nanogap electrodes in high-resolution patterning. To demonstrate the quality of the fabricated nanogap, we measured a single electron tunneling through the dodecanethiol-coated Au nanoparticles placed in a nanogap.

Figure 1 shows a schematic drawing of the fabrication process using the conventional EBL and the SAM lithographic techniques. First, the EBL followed by metal deposition and lift-off process is used to make the first metal electrode on a silicon oxide layer on a $\mathrm{Si}$ wafer, as shown in Fig. 1(a). Next, the multilayer films composed of a functional mercaptoalkanoic acid [16-mercaptohexadecanic acid (MHDA), Aldrich] and a coordinated copper ion [inorganic salt copper(II) perchlorate, Aldrich] are formed layer by layer on the surface of the first metal electrode. ${ }^{18}$ by following an established procedure ${ }^{13-16}$ [Fig. 1(b)]. Especially, we took a longer time $(72 \mathrm{~h})$ for the first immersion in MHDA in the construction of the multilayer films, since a short immersion time of several minutes leads to create a defect-rich SAM. The EBL and sequent metal deposition are carried out to fabricate the second electrode on the surface [Fig. 1(c)]. Finally, the multilayer films are chemically removed by immersion in a photoresist stripper (ACT935J, Air Products and Chemicals, Inc.) at $40{ }^{\circ} \mathrm{C}$ for $15 \mathrm{~min}$ after sonication for $1 \mathrm{~min}$ in ethanol [Fig. 1(d)]. Two metal electrodes with nanogaps whose size is precisely defined by the molecular ruler are formed in the high-resolution patterning by using the EBL. Using the method, we obtained the yield of nanogap electrodes with a fine structure more than $80 \%$.

Figure 2(a) is a scanning electron microscope (SEM) image showing a typical nanogap fabricated using this method. Since the nanogap was formed using only a single layer of molecular resist, the gap size was expected to be 
(a)

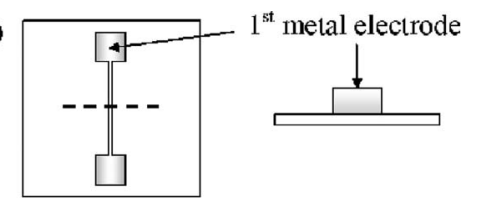

(b)

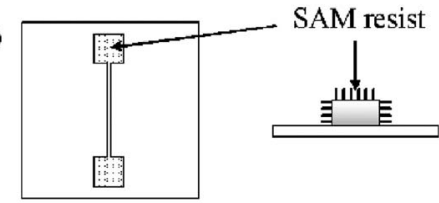

(c)

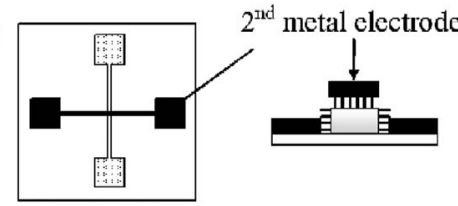

(d)

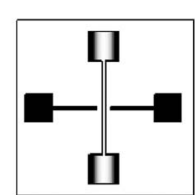

FIG. 1. Schematic drawing of fabrication process. (a) EBL is used to make the first metal electrode and the metal layer is deposited by sputtering. (b) Multilayer films are formed layer by layer on surface of the first electrode. (c) The EBL is repeated. (d) Multilayer films are chemically removed by immersion in a photoresist stripper. The nanogaps are formed between the two metal electrodes.

$2 \mathrm{~nm}$. To confirm this, we estimated the gap size from its current-voltage $(I-V)$ characteristic because measuring the size of the gap accurately with subnanometer resolution by SEM is difficult. Figure 2(b) shows the $I-V$ curve obtained with the gap shown in Fig. 2(a). The solid line indicates a fitting curve to the $I-V$ data measured, which was achieved using the Simmons tunneling model. ${ }^{20}$ In the model, the tunneling current is described as

$$
I=\frac{k_{1} A}{s^{2}}\left[x^{2} \exp \left(-k_{2} s x\right)-y^{2} \exp \left(-k_{2} s y\right)\right],
$$

where $x=\sqrt{\phi-V / 2}, y=\sqrt{\phi+V / 2}, k_{1}=6.32 \times 10^{10} \mathrm{~V} \mathrm{~s}^{-1}$, and $k_{2}=1.025 \mathrm{~J}^{-1 / 2}$. The $s, A$, and $\phi$ stand for the gap size, the emission area, and the barrier height, respectively. From the fitting curve, we obtained the parameters of $A=5.1$ $\times 10^{-17} \mathrm{~cm}^{2}$ and $\phi=0.16 \mathrm{eV}$, which are reasonable values compared with the results obtained with a gap of approximately $1 \mathrm{~nm}^{6}$ Moreover, the estimated gap size $s=2.2 \mathrm{~nm}$ agrees well with the film thickness of the multilayer resist

(a)

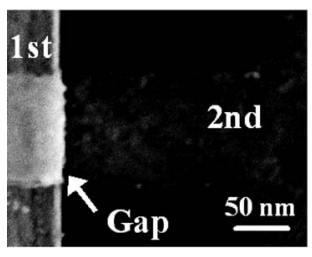

(b)

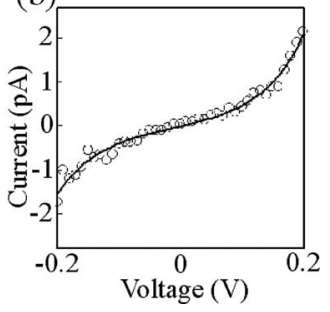

FIG. 2. (a) SEM image of nanogap fabricated between two metal electrodes. Nanogap was formed using a single-layer molecular resist. (b) Currentvoltage data obtained at the nanogap device as shown in (a). The solid line indicates a fit to the data measured, which was achieved using the Simmons

nanoparticle is located near one side of the electrode because
Downloaded 19 Jul 2010 to 133.1.91.151. Redistribution subject to AIP license or copyright; see http://apl.aip.org/apl/copyright.jsp

(a)
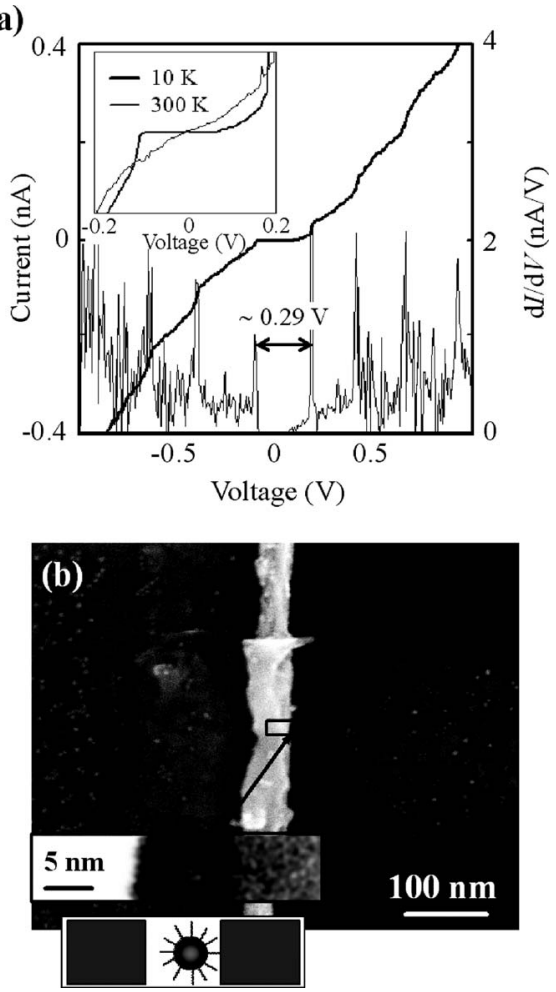

FIG. 3. (a) Current-voltage curves obtained at the nanogap device after the dispersion of $\mathrm{Au}$ nanoparticles. The Coulomb staircase is clearly observed. Enlarged graph near $V=0 \mathrm{~V}$ is also shown in the inset. (b) SEM image of the nanogap electrodes from which the $I-V$ curve shown in (a) was taken. The single nanoparticle was placed to make an asymmetric junction between the two electrodes.

(about $2 \mathrm{~nm}$ ). This result suggests that the gap size is precisely defined by the film thickness of the multilayer resist.

To determine the accuracy of the gap size and the quality of the formed gap, we also measured the electronic transport property of dodecanethiol $\left(\mathrm{CH}_{3}\left(\mathrm{CH}_{2}\right)_{11} \mathrm{SH}\right)$-coated Au nanoparticles. The preparation of nanoparticles followed an established procedure by Santhanam et al. ${ }^{19}$ The citrate-stabilized $\mathrm{Au}$ sol with a normal size of $5 \mathrm{~nm}$ required for the procedure was purchased from British Biocell International Inc. The dodecanethiol-coated Au nanoparticles were dispersed on the nanogap devices by spin casting at $1000 \mathrm{rpm}$ for $60 \mathrm{~s}$. The isolated single nanoparticles can be placed in nanogaps through the dispersion process under the condition of the spinner. The diameter of the gold nanoparticles including the dodecanethiol is approximately $7.8 \mathrm{~nm}$ and the size of the designed nanogap was approximately $10 \mathrm{~nm}$ using five layers of the multilayer film. Before the dispersion of the $\mathrm{Au}$ particles on the nanogap device, we observed no tunneling current in the range of the applied voltage from -0.5 to $0.5 \mathrm{~V}$. This result means that the two electrodes were sufficiently isolated. After the dispersion of the Au nanoparticles, a Coulomb staircase was clearly observed at $T=10 \mathrm{~K}$, as indicated by the thick line in Fig. 3(a). We can estimate the Coulomb gap voltage to be approximately $0.29 \mathrm{~V}$ from the differential conductance $(d I / d V)$. The Coulomb staircase disappeared at $T=300 \mathrm{~K}$ (thin line) due to the increase of the thermal effect, as shown in the inset of Fig. 3(a).

Figure 3(b) shows a SEM image of the nanogap device by which the $I-V$ curve in Fig. 3(a) was obtained. The Au nanoparticle is located near one side of the electrode because Aicense or copyright; see http://apl.aip.org/apl/copyright.jsp 
the gap is slightly larger than the nanoparticle. The relationship between the asymmetric property shown in Fig. 3(b) and the $I-V$ curve shown in Fig. 3(a) is consistent with the theoretical reports: theoretical calculations have predicted that the $I-V$ curves obtained at an asymmetric and a symmetric junction show the Coulomb staircase and the monotonic rise shapes, respectively, with the bias beyond the Coulomb gap voltage. ${ }^{21,22}$

Finally, we determined whether the measured Coulomb gap voltage was a reasonable value for the single electron tunneling through a nanoparticle with a size of $5 \mathrm{~nm}$. We can calculate the $C_{p}$ capacitance of a sphere conductor to be $5.6 \times 10^{-19} \mathrm{~F}$ by modeling the nanoparticle-electrode junction using the image charge method, ${ }^{23}$ and thus we obtain the Coulomb gap voltage $V_{c}=e / C_{p}=0.32 \mathrm{~V}$, which agrees well with our experimental results $\left(V_{c}=\right.$ approximately $\left.0.29 \mathrm{~V}\right)$.

In summary, we developed and tested a new method of fabricating nanogaps using a combination of the EBL and the SAM lithographic techniques. This method enables us to control the size of a gap with an accuracy of approximately $2 \mathrm{~nm}$ as well as to control the position and the width of the gap electrodes in high-resolution patterning by using EBL. As a demonstration, we investigated the electronic transport property of dodecanethiol-coated Au nanoparticles using the nanogap devices. The $I-V$ curve showed a clear Coulomb staircase as expected because we fabricated the nanogap to be an asymmetric junction by controlling the gap size. Our new method should be useful in fabricating functional nanodevices. $^{24}$

The authors would like to thank Dr. W. Huang of the Institute for Molecular Science (IMS), Japan for supporting experiment on the $I-V$ measurements. The authors also thank H. Ozawa of the IMS for preparing the dodecanethiol-coated Au nanoparticles. A part of this work was supported by the Nanotechnology Support Project of the Ministry of Education, Culture, Sports, Science and Technology (MEXT), Japan.
${ }^{1}$ A. Bezryadin, C. Dekker, and G. Schmid, Appl. Phys. Lett. 71, 1273 (1997).

${ }^{2}$ K. Liu, Ph. Avouris, J. Bucchignano, R. Martel, S. Sun, and J. Michl, Appl. Phys. Lett. 80, 865 (2002).

${ }^{3}$ H. Park, J. Park, A. K. L. Lim, E. H. Anderson, A. P. Alivisatos, and P. L. McEuen, Nature (London) 407, 57 (2000).

${ }^{4}$ D. Porath, A. Bezryadin, S. de Vries, and C. Dekker, Nature (London) 403, 635 (2000).

${ }^{5}$ A. Bachtold, P. Hardly, T. Nakanishi, and C. Dekker, Science 294, 1317 (2001).

${ }^{6}$ P. Steinmann and J. M. R. Weaver, Appl. Phys. Lett. 86, 063104 (2005). ${ }^{7}$ G. Philipp, T. Weimann, P. Hinze, M. Burghard, and J. Weis, Microelectron. Eng. 46, 157 (1999).

${ }^{8}$ Y. Naitoh, K. Tsukagoshi, K. Murata, and W. Mizutani, e-J. Surf. Sci. Nanotechnol. 1, 41 (2003).

${ }^{9}$ H. Park, A. K. L. Lim, A. P. Alivisatous, J. Park, and P. L. McEuen, Appl. Phys. Lett. 75, 301 (1999).

${ }^{10}$ A. M. Morpurgo, C. M. Marcus, and D. B. Robinson, Appl. Phys. Lett. 74, 2084 (1999)

${ }^{11}$ S. Boussaad and N. J. Tao, Appl. Phys. Lett. 80, 2398 (2002).

${ }^{12}$ M. A. Reed, C. Zhou, C. J. Muller, T. P. Burgin, and J. M. Tour, Science 278, 252 (1997)

${ }^{13}$ A. Hatzor and P. S. Weiss, Science 291, 1019 (2001).

${ }^{14}$ M. E. Anderson, R. K. Smith, Z. J. Donhauser, A. Hatzor, P. A. Lewis, L. P. Tan, M. W. Horn, and P. S. Weiss, J. Vac. Sci. Technol. B 20, 2739 (2002).

${ }^{15}$ M. E. Anderson, L. P. Tan, H. Tanaka, M. Mihok, H. Lee, M. W. Horn, and P. S. Weiss, J. Vac. Sci. Technol. B 21, 3116 (2003).

${ }^{16}$ H. Tanaka, M. E. Anderson, M. W. Horn, and P. S. Weiss, Jpn. J. Appl. Phys., Part 1 43, L950 (2004).

${ }^{17}$ G. S. McCarty, Nano Lett. 4, 1391 (2004).

${ }^{18}$ S. D. Evans, A. Ulman, K. E. Goppert-Berarducci, and J. L. Gerenser, J. Am. Chem. Soc. 113, 5866 (1991).

${ }^{19}$ V. Santhanam, J. Liu, R. Agarwal, and R. P. Andres, Langmuir 19, 7881 (2003).

${ }^{20}$ J. G. Simmons, J. Appl. Phys. 34, 1793 (1963).

${ }^{21}$ M. Amman, R. Wilkins, E. Ben-Jacob, P. D. Marker, and R. C. Jaklevic, Phys. Rev. B 43, 1146 (1991).

${ }^{22}$ A. E. Hanna and M. Tinkham, Phys. Rev. B 44, 5919 (1991).

${ }^{23}$ C. Gao, F. Duewer, and X. D. Xiang, Appl. Phys. Lett. 75, 3005 (1999).

${ }^{24} \mathrm{~N}$. Asahi, M. Akazawa, and Y. Amemiya, IEEE Trans. Electron Devices 42, 1999 (1995) 